\title{
Limitations in Electrophysiological Model Development and Validation Caused by Differences between Simulations and Experimental Protocols
}

\author{
Jesús Carro ${ }^{\mathrm{a}, \mathrm{b}, \mathrm{c}, *}$, José F. Rodríguez-Matas $^{\mathrm{d}, \mathrm{b}}$, Violeta Monasterio ${ }^{\mathrm{a}}$, \\ Esther Pueyo ${ }^{b, c}$ \\ ${ }^{a}$ Universidad San Jorge. Campus Universitario, Autov A23 km 299, 50830. Villanueva de \\ Gállego Zaragoza. SPAIN \\ ${ }^{b}$ Aragon Institute for Engineering Research, Universidad de Zaragoza, SPAIN \\ ${ }^{c}$ CIBER in Bioengineering, Biomaterials \& Nanomedicine (CIBER-BBN), SPAIN \\ ${ }^{d}$ LaBS, Department of Chemistry, Materials and Chemical Engineering "Giulio Natta", \\ Politecnico di Milano, ITALY
}

\begin{abstract}
Models of ion channel dynamics are usually built by fitting isolated cell experimental values of individual parameters while neglecting the interaction between them. Another shortcoming regards the estimation of ionic current conductances, which is often based on quantification of Action Potential (AP)-derived markers. Although this procedure reduces the uncertainty in the calculation of conductances, many studies evaluate electrophysiological AP-derived markers from single cell simulations, whereas experimental measurements are obtained from tissue preparations. In this work, we explore the limitations of these approaches to estimate ion channel dynamics and maximum current conductances and how they could be overcome by using multiscale simulations of experimental protocols.

Four human ventricular cell models, namely ten Tusscher and Panfilov (2006), Grandi et al. (2010), O’Hara et al. (2011), and Carro et al. (2011), were used. Two problems involving scales from ion channels to tissue were investigated: 1) characterization of L-type calcium voltage-dependent inactivation $\left.\left(I_{C a, L}\right) ; 2\right)$

\footnotetext{
* Corresponding author

Email addresses: jcarro@usj.es (Jesús Carro ), josefelix.rodriguezmatas@polimi.it (José F. Rodríguez-Matas), vmonasterio@usj.es (Violeta Monasterio), epueyo@unizar.es (Esther Pueyo)
} 
identification of major ionic conductance contributors to steady-state AP markers, including $A P D_{90}, A P D_{75}, A P D_{50}, A P D_{25}$, Triangulation and maximal and minimal values of $V$ and $d V / d t$ during the $\operatorname{AP}\left(V_{\max }, V_{\min }, d V / d t_{\max }\right.$, $\left.d V / d t_{\min }\right)$.

Our results show that: 1) $I_{C a, L}$ inactivation characteristics differed significantly when calculated from model equations and from simulations reproducing the experimental protocols. 2) Large differences were found in the ionic currents contributors to $A P D_{25}$, Triangulation, $V_{\max }, d V / d t_{\max }$ and $d V / d t_{\min }$ between single cells and 1D-tissue.

When proposing any new model formulation, or evaluating an existing model, consistency between simulated and experimental data should be verified considering all involved effects and scales.

Keywords: Cardiac Modeling, Ionic currents, Electrophysiology, Action potential, Model validation 


\section{Introduction}

From the earliest mathematical model of an electrical cell's action potential (AP) developed by Hodgkin and Huxley in the fifties, the complexity of current AP models has grown considerably. The advent of new experimental

5 techniques has made large sets of experimental data readily available, which has motivated the development of more complex models to accurately describe cellular electrical activity. Whereas growing in model complexity is a natural consequence of the increased knowledge (Noble et al., 2012), the more complex the model, the more difficult the identification of model parameters tends to be. An AP model involves the sum of different transmembrane ionic currents and the balance between intra- and extra-cellular ionic concentrations. Each ionic current follows a mathematical formulation in which several effects are present, e.g., ion channel activation and inactivation gating or current conductance. For each effect, a number of model parameters are identified based on data from experimental protocols specific for each particular ionic current.

The experimental protocols used to obtain most of the parameters of each ionic current are performed in isolated cells. But, due to the sensitivity of some ionic channels to the cell isolation process used in voltage-clamp experiments (Yue et al., 1996), the conductances of the ionic currents in cardiac models are often not estimated from direct measurements of the current density. Instead, individual channel conductances are adjusted so that measures from model-generated APs closely match experimental AP measurements in tissue such as AP duration (APD) or others. In the Courtemanche-Ramirez-Nattel $(\mathrm{CRN})$ model (Courtemanche et al. 1998), the ionic conductances $G_{N a}, G_{K 1}$, ${ }_{25} G_{t o}, G_{K r}$ and $G_{K s}$ were fitted to obtain a correct input resistance, AP morphology, AP amplitude (APA) and upstroke velocity $\left(d V / d t_{\max }\right)$. In a late version of the Luo-Rudy (LR) model (Zeng et al. 1995), $G_{K s}$ was fitted to get the right APD prolongation when the $I_{K s}$ current was blocked. Taking those models as an example, in the tenTusscher-Noble-Noble-Panfilov (TNNP04) model 30 (ten Tusscher et al., 2004), $G_{K s}$ was set to obtain physiologically plausible APD 
values for each cell type (epicardial, midmyocardial and endocardial). In the Grandi-Pascualini-Bers (GPB) model (Grandi et al. 2010), $G_{N a}$ was set so as to reproduce experimental measurements of APA and maximum value of the transmembrane potential $\left(V_{\max }\right)$. In the O'Hara-Rudy dynamic (ORd) model (O'Hara et al., 2011), the potassium current conductances were fitted to reproduce the experimental effect on the APD when they were blocked. Finally, in the Carro-Rodríguez-Laguna-Pueyo (CRLP) model (Carro et al., 2011), using the sensitivity analysis proposed in Romero et al. (2009), $G_{K 1}, G_{N a K}, G_{C a, L}$, and $G_{N a}$ were fitted to obtain not only APD values within physiological ranges, but also other markers of arrhythmic risk, including time constants of APD rate adaptation or rate dependence of ionic concentrations.

On the contrary, the parameters that model current kinetics (gating parameters) are usually identified from single-cell experiments. The calibration process is usually performed using a nonlinear least square fitting of voltage clamp data

45 by assuming that each parameter's effect is independent from the rest (e.g., the steady-state of an inactivation gate is calibrated against experimental results while considering that the time constant of the gate does not affect such results, which might not be correct). . However, when the complexity of the model increases, the interaction between effects becomes increasingly important. Therefore, assuming independence of the effects when identifying model parameters may be misleading. While other techniques have been proposed in recent years to improve the fitting of the gating parameters (Csercsik et al. 2012: Dokos and Lovell, 2004, Lee et al., 2006; Wang and Beaumont, 2004), none of the models analyzed in the present study have used such techniques.

Once model parameters have been identified, the resulting AP models are validated against experimental measurements commonly obtained also from tissue preparations. Characteristics such as resting membrane potential $\left(V_{\min }\right)$ and upstroke velocity $\left(d V / d t_{\max }\right)$ are usually compared between model-generated and experimental APs. In the CRN model, the role of different ionic conductances, the morphology of the AP, and the behavior of the model under different cycle lengths (CLs) were compared with experimental observations. In the 
updated version of the LR model (Zeng et al. 1995), the theoretical APD restitution curve was compared with an experimental restitution curve obtained by means of optical recordings of cardiac APs. In the TNNP04 model, simulated 65 APD restitution curves (at $90 \%$ repolarization, $A P D_{90}$ ) were evaluated in single cells to validate the model against experimental results measured in tissue preparations. Also in this model, propagation in a homogeneous one-dimensional (1D) tissue was simulated to validate the model in terms of Conduction Velocity (CV). In a subsequent version of the model, the ten Tusscher-Panfilov 70 (TP06) model (ten Tusscher and Panfilov, 2006), simulated APD restitution curves (at $90 \%$ and $50 \%$ repolarization) in single cells were compared with experimental results. The GPB model was validated by comparing the predicted $A P D_{90}$ prolongation caused by blockade of different potassium currents with experimental results. The CRLP model, as the GPB model, was validated by comparing $A P D_{90}$ prolongation caused by potassium current blockades with experimental results, but also by comparing a number of computed markers not used in the fitting process.

For the aforementioned reasons, problems appear in the calibration and/or validation of electrophysiological models caused by two related situations: submodel variable interactions and cell-to-cell interactions during the AP propagation. Parameters related to ion channel gating kinetics are commonly obtained by considering each gate of the channel independently. Ionic conductances are adjusted or validated with experimental data obtained from tissue preparations by using single cell computer simulations. In both situations, the differences caused by not considering the corresponding interactions introduce non-negligible cross-effects between parameters that are not considered in the fitting process.

Other studies have analyzed problems related to the two issues aforementioned in this work. Cherry and Fenton (2007) analyzed the differences between models of the same species to represent electrophysiological properties and how these differences propagate to tissue simulations. Pathmanathan et al. (2015) studied how the uncertainty in the definition of the gating variables propagates 
in multi-scale models and highlighted the need to use observations across multiple scales. Shotwell and Gray (2016) analyzed the problems caused by the use of observations across multiple scales and how to characterize the relationships between the model parameters and the effect that they have in the multi-scale model outputs.

In this work, experimental protocols are simulated in silico to analyze the consequences of the corresponding interactions in two scenarios involving scales ranging from ion channels to tissue: 1) characterization of L-type calcium voltage-dependent inactivation; 2) identification of ionic current conductances with the largest contribution to steady-state AP markers. In the first case, differences between the mathematical model, simulation results and experimental measurements are analyzed to evaluate how interactions affect the development and validation of mathematical ion channel models. In the second case, differences between AP markers simulated in isolated cells and in homogeneous $1 \mathrm{D}$ tissue are analyzed to evaluate how propagation affects their values and to assess the importance of each ionic current on each marker.

\section{Materials and Methods}

\subsection{Human Ventricular Cell Models}

In AP models, ionic currents are controlled by activation and inactivation gates. Gates are modeled by functions varying between 0 and 1 . Below a threshold potential, the activation gates are closed (the function value is 0 ) and the inactivation gates are open (value 1 ). When the transmembrane potential increases, the activation gates open (their values increase towards 1) and the inactivation gates close (their values decrease towards 0 ). In particular for the $I_{C a, L}$ current, all human ventricular cell models analyzed in this study have one voltage-dependent activation gate $d$. This activation gate $d$ multiplies the expression for the inactivation gating, whose formulation differs greatly between models. For this work, we selected four of the most recently developed human ventricular cell models: the GPB model, the TP06 model, the ORd model and 
the CRLP model. In these models we studied the voltage-dependent inactivation of the L-type calcium current.

The TP06 model is one of the most extensively used ventricular AP models. It is an improved version of the model published in 2004 (ten Tusscher et al. 2004) in which the calcium dynamics, the slow delayed rectifier potassium current $\left(I_{K s}\right)$ and the $I_{C a, L}$ were reformulated. The TP06 model is based on experimental data from human cardiomyocytes for most ionic currents and is defined for three types of cells: epicardial, midmyocardial and endocardial. In the TP06 model, voltage-dependent $I_{C a, L}$ inactivation is modeled as the product of a fast, $f_{2}$, and a slow, $f$, voltage-dependent inactivation gates as well as a calcium-dependent inactivation gate, $f_{C a}$ :

$$
\operatorname{Inact}_{C a, L}^{T P 06}=f \cdot f_{2} \cdot f_{C a}
$$

These gates have different time constants $\left(\tau_{f_{2}}, \tau_{f}\right.$ and $\left.\tau_{f_{C a}}\right)$ and steady-state values $\left(f_{2, \infty}, f_{\infty}\right.$ and $\left.f_{C a, \infty}\right)$. This formulation is based on experiments that indicate the presence of both fast and slow voltage-dependent $I_{C a, L}$ recovery process (Li and Nattel, 1997; Magyar et al., 2002, Pelzmann et al., 1998).

The GPB model is based on the rabbit AP model proposed in Shannon et al. (2004), which includes subsarcolemmal and junctional compartments in the formulation of the currents and provides a detailed description of calcium handling. The GPB model includes new definitions of ionic current densities and kinetics and is defined for endocardial and epicardial cells. In the GPB model, voltagedependent $I_{C a, L}$ inactivation is modeled by a single voltage-dependent gate $f$ and two calcium-dependent gates, one for the subsarcolemmal compartment, $f_{C a, s l}$, and another one for the junctional compartment, $f_{C a, j}$. The formulation of $I_{C a, L}$ inactivation in the GPB model is as follows:

$$
\operatorname{Inact}_{C a, L}^{G P B}=f \cdot\left(A_{j} \cdot f_{C a, j}+A_{s l} \cdot f_{C a, s l}\right)
$$

where $A_{j}$ and $A_{s l}$ are the ratios of calcium-dependent inactivation channels in the junctional and the subsarcolemmal compartment, respectively $\left(A_{j}+A_{s l}=\right.$ $1301)$. 
The CRLP model is a modification of the GPB model. As in the original model, the modified CRLP model describes the AP of endocardial and epicardial human ventricular myocytes. The CRLP model reformulates $I_{C a, L}$, readjusts the parameters of $I_{K 1}$ and redefines a number of model parameters, including $G_{N a}$ (maximum $I_{N a}$ conductance) and $G_{N a K}$ (maximal $I_{N a K}$ value). In the CRLP model, the formulation of voltage-dependent $I_{C a, L}$ inactivation is similar to that of the TP06 model. However, the associated time constants were adjusted so as to better reflect the adaptation of the APD to CL changes. The calciumdependent gates were maintained as in the original GPB model. The formulation of $I_{C a, L}$ inactivation in the CRLP model is as follows:

$$
\operatorname{Inact}_{C a, L}^{C R L P}=f \cdot f_{2} \cdot\left(A_{j} \cdot f_{C a, j}+A_{s l} \cdot f_{C a, s l}\right)
$$

The ORd model is the most recent human ventricular AP model. In their work, O'Hara and coworkers (O'Hara et al. 2011), propose an AP model based on extensive undiseased human ventricular data. Epicardial, midmyocardial and endocardial models were developed by using human mRNA and protein data. The ORd model has the most complex definition of $I_{C a, L}$ of all studied AP models. $I_{C a, L}$ inactivation is modeled as a weighted average involving voltagedependent gates. The gates $f_{f}$ and $f_{s}$ represent the behavior of the voltagedependent gates when there is no calcium. The gates $f_{C a, f}, f_{C a, s}$ and $j_{C a}$ represent the behavior of the voltage-dependent gates when calcium is present. The calcium-dependent gate $n$ modulates the relative weight of both families of gates. The $n$ gate is modeled as a Markov chain. The gates $f_{f}, f_{s}, f_{C a, f}$, $f_{C a, s}$ and $j_{C a}$ have different time constants, $\tau_{f, f}, \tau_{f, s}, \tau_{f, C a, f}, \tau_{f, C a, s}$ and $\tau_{j}$, but the same steady-state value $f_{s s}$. As discussed by Thomas O'Hara in the online version of the article, there are some issues related to the $I_{N a}$ current formulation in the model. The authors propose to replace the $I_{N a}$ current of the original ORd model with the formulation proposed in the TP06 model. In this work we analyze the original ORd model and the ORd model with the $I_{N a}$ current of the TP06 model (ORdNa). The formulation of $I_{C a, L}$ inactivation in 
the ORd and ORdNa models is as follows:

$\operatorname{Inact}_{C a, L}^{O R d}=\left(A_{f, f} \cdot f_{f}+A_{f, s} \cdot f_{s}\right) \cdot(1-n)+j_{C a} \cdot\left(A_{f, C a, f} \cdot f_{C a, f}+A_{f, C a, s} \cdot f_{C a, s}\right) \cdot n$ where $A_{f, f}$ and $A_{f, C a, f}$ are, respectively, the ratios of the fast voltage-dependent inactivation gates $f_{f}$ and $f_{C a, f}$, and $A_{f, s}$ and $A_{f, C a, s}$ are, respectively, the ratios of the slow voltage-dependent inactivation gates $f_{s}$ and $f_{C a, s}$.

\subsection{Characterization of L-type calcium voltage-dependent inactivation}

Voltage-dependent $I_{C a, L}$ inactivation is experimentally characterized using the paired-pulse protocol, which is illustrated in Figure1. This protocol consists of clamping the membrane potential from a holding potential $V_{\text {hold }}$ to different pre-pulse potentials $V_{\text {pre }}$ during a specified time interval $t_{\text {pre }}$. After this time, the potential is clamped to a pulse potential $V_{\text {pulse }}$ during an interval $t_{\text {pulse }}$. Following this, the membrane potential is clamped back to $V_{\text {hold }}$. Variations of this protocol introduce a separation between the pre-pulse and the pulse potential, during which the potential is set to $V_{\text {hold }}$ for a short period of time, $t_{\text {sep }}$, before clamping the membrane potential to $V_{\text {pulse }}$. Steady-state voltagedependent $I_{C a, L}$ inactivation is obtained during the $t_{p u l s e}$ interval. In this part of the test, peak $I_{C a, L}$ current is measured for each potential used as pre-pulse and results are normalized by the peak value of the current measured for the minimum pre-pulse potential. The resulting curve is used in the models as the steady-state value of the voltage-dependent inactivation $\left(f_{s s}\right)$.

Human ventricular cell models use different experimental datasets to define their voltage-dependent $I_{C a, L}$ inactivation functions. With the aim of comparing the simulation results of steady-state $I_{C a, L}$ inactivation with experimental results, we selected the same experimental dataset that was used to adjust each model in the original articles. The TP06 model does not indicate which experimental results were used to fit the steady-state voltage-dependent $I_{C a, L}$ inactivation, whereas in the previous version of the model (ten Tusscher et al. 2004) they use data from Pelzmann et al. (1998). For the TP06 model the authors redefine the $I_{C a, L}$ expression by adding a second voltage-dependent inactivation gate. In 


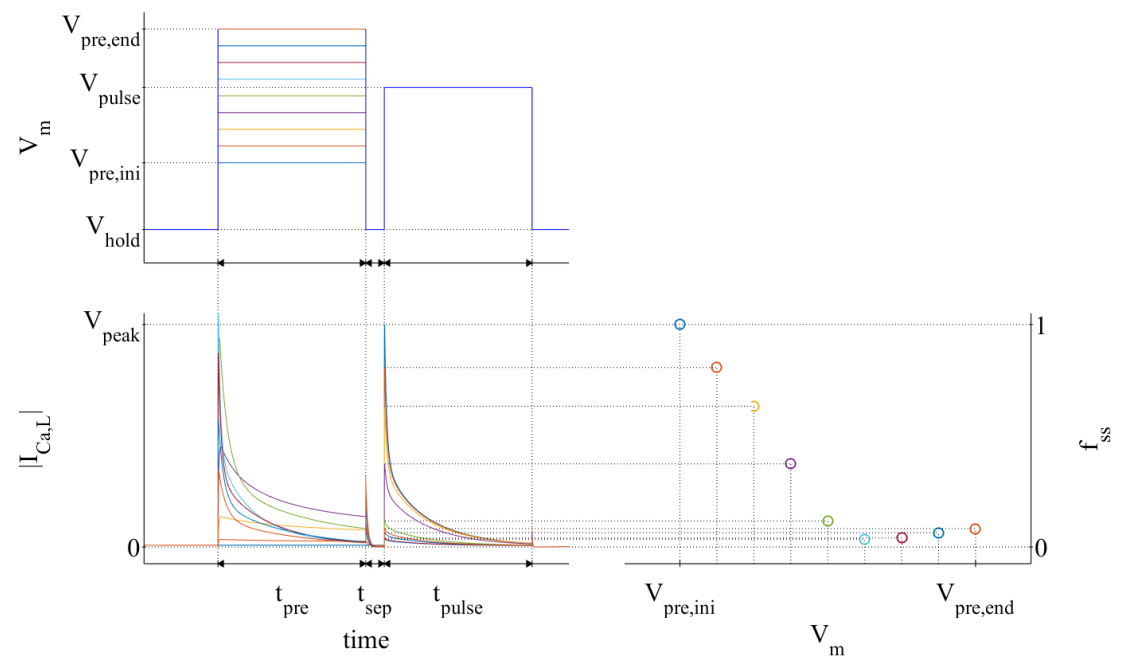

Figure 1: Paired-pulse voltage clamp protocol and measurement of $f_{s s}$

addition, only the $I / V$ curve for $I_{C a, L}$ is compared with experimental results from Magyar et al. (2000). The GPB model uses experimental results from Li et al. (1999) and the ORd model from Magyar et al. (2000).

Each set of experimental results uses a different configuration of the pairedpulse protocol to characterize the steady-state voltage-dependent $I_{C a, L}$ inactivation (Table 1). Also, different calcium concentrations in the extracellular solution and in the patch pipette solution, varying from 1.8 to $5.4 \mathrm{mM}$ and from 0 to $2.0 \mathrm{mM}$, respectively, are used in each experimental dataset.

In our work we performed in silico simulations in which we recreated the experimental paired-pulse tests for $I_{C a, L}$ characterization. Intracellular and extracellular concentrations were set at the values used in the experimental protocols. In particular, free intracellular calcium $\left(\left[\mathrm{Ca}^{2+}\right]_{i}\right)$ was set as the value of the pipette solutions of the experiments. To do this, we fixed the value by setting its derivative to zero $\left(d\left[\mathrm{Ca}^{2+}\right]_{i} / d t=0\right)$. All the calcium buffers and the diffusion and transportation of calcium inside the cell were maintained as in the original models. This allowed calcium concentrations in all remaining intracellu- 


\begin{tabular}{|c|c|c|c|}
\hline & $\frac{\text { Pelzmann et al. }}{(1998)}$ & Li et al. (1999) & $\frac{\text { Magyar et al. }}{(2000)}$ \\
\hline$V_{\text {hold }}(m V)$ & -45 & -80 & -80 \\
\hline$V_{\text {pulse }}(m V)$ & 10 & 10 & 5 \\
\hline$V_{\text {pre }, \text { ini }}(m V)$ & -40 & -100 & -55 \\
\hline$V_{\text {pre,fin }}(m V)$ & 40 & 60 & 15 \\
\hline$\Delta V_{\text {pre }}(m V)$ & 5 & 10 & 5 \\
\hline$t_{\text {pre }}(m s)$ & 400 & 400 & 500 \\
\hline$t_{\text {sep }}(m s)$ & 10 & 5 & 0 \\
\hline$t_{\text {pulse }}(m s)$ & 400 & 300 & 390 \\
\hline
\end{tabular}

Table 1: Paired-pulse test parameters in experimental protocols.

lar compartments to follow the dynamics described in the corresponding model equations. The same values for $V_{\text {hold }}, V_{\text {pre }}$, and $V_{\text {pulse }}$ as in the corresponding experiments (see Table 1) were used. All currents except for $I_{C a, L}$ were blocked.

\subsection{Steady-state AP markers}

To study the effect of considering data measured at tissue level for characterizing single cell models, AP markers calculated at single cell and tissue levels with the four ventricular models were compared. The following markers were analyzed:

- Action Potential Duration (APD): APD is considered the main preclinical marker of drug cardiotoxicity. APD prolongation has been linked to long QT syndrome and increased risk for Torsades de Pointes (Hondeghem et al., 2001, Volders et al., 2000). In this study we measured the APD at different percentages of repolarization $(90 \%, 75 \%, 50 \%, 25 \%)$.

- Triangulation: This marker quantifies the shape of the final part of the AP and is calculated as the difference between APD at $50 \%$ and $90 \%$ repolarization. Low triangulation values indicate square APs, while high 
values indicate triangular APs. Triangulation has been proposed as a marker of pro-arrhythmia (Hondeghem et al., 2001).

- Minimum and Maximum Transmembrane Potential and Transmembrane Potential Velocity: Electrophysiological changes at the cellular level can cause disorders in the minimum and maximum values of the membrane potential. For example, under hyperkalemic conditions, the resting potential is increased from $-85 \mathrm{mV}$ to $-60 \mathrm{mV}$, the maximum potential falls, and the upstroke may be subdivided into more than one component (Carmeliet, 1999). The maximum value of the AP is used in many models to adjust the cardiac conductances (Courtemanche et al. 1998 Grandi et al., 2010) whereas the minimum potential is used for model validation (O'Hara et al. 2011).

\subsection{Ionic contributors to AP markers}

To evaluate the role played by each ionic current in determining each physiological marker, the results of the simulations were adjusted by a first order response surface model:

$$
M_{j} \approx C_{j}+\sum_{i=1}^{N} \Delta_{i} \cdot m_{j, i}
$$

where $M_{j}$ is the value of the physiological marker $j$ under the analyzed condition, $C_{j}$ is the value of the marker $\mathrm{j}$ under control conditions, $m_{i, j}$ is the weight of the current $i$ in contributing to the marker $j$ and $\Delta_{i}$ is the variation of the ionic conductance:

$$
\Delta_{i}=\frac{G_{i}-G_{i, 0}}{G_{i, 0}}
$$

where $G_{i, 0}$ and $G_{i}$ are the values of the ionic conductance at control and at the condition under analysis, respectively.

For each individual marker, if we concatenate the results for the different evaluated conditions $k=1, \ldots, K$, this system can be expressed in matrix form 
as:

$$
\begin{gathered}
{\left[\begin{array}{c}
M_{j, 1} \\
M_{j, 2} \\
\vdots \\
M_{j, K}
\end{array}\right] \approx\left[\begin{array}{cccc}
\Delta_{1,1} & \Delta_{2,1} & \cdots & \Delta_{N, 1} \\
\Delta_{1,2} & \Delta_{2,2} & \cdots & \Delta_{N, 2} \\
\vdots & \vdots & \ddots & \vdots \\
\Delta_{1, K} & \Delta_{2, K} & \cdots & \Delta_{N, K}
\end{array}\right] \cdot\left[\begin{array}{c}
m_{j, 1} \\
m_{j, 2} \\
\vdots \\
m_{j, K}
\end{array}\right]+C_{j} \cdot\left[\begin{array}{c}
1 \\
1 \\
\vdots \\
1
\end{array}\right] \rightarrow} \\
\rightarrow \bar{M}_{j} \approx \overline{\bar{\Delta}} \cdot \bar{m}_{j}+C_{j} \cdot \bar{J}_{K, 1}
\end{gathered}
$$

where $M_{j, k}$ is the value of the marker $j$ and $\Delta_{i, k}$ is the variation of the ionic conductance $i$ at condition $k$. Using this notation, the weights of the ionic conductances can be calculated as follows:

$$
\bar{m}_{j}=\left(\overline{\bar{\Delta}}^{T} \cdot \overline{\bar{\Delta}}\right)^{-1} \cdot \overline{\bar{\Delta}}^{T} \cdot\left(\bar{M}_{j}-C_{j} \cdot \bar{J}_{K, 1}\right)
$$

\subsection{Computational simulations}

The models were stimulated with square current pulses with an amplitude of twice the diastolic threshold and a duration of $1 \mathrm{~ms}$. Depending on the simulation scale, the diastolic threshold was defined as the minimum amplitude required to (a) generate five APs (single cell simulations) or (b) propagate five APs (1D tissue simulations).

To simulate steady-state conditions, models were stabilized with a train of 100 stimulations at a CL of $1000 \mathrm{~ms}$. Electrophysiological markers were calculated from the last simulated beat. Every model was first simulated under control conditions and then each ionic conductance was varied by \pm 15 and $\pm 30 \%$.

For 1D tissue simulations, a homogeneous 3-cm long fiber composed of epicardial cells was used. The value of the conductance, $\sigma$, was set to obtain a Conduction Velocity (CV) close to $65 \mathrm{~cm} / \mathrm{s}$ (Taggart et al., 2000). The cell capacitance was set to $C_{m}=1 \mu \mathrm{F} / \mathrm{cm}^{2}$. The AP markers were computed as the mean value of the markers measured at five different positions within the cable located at: $1,1.25,1.5,1.75$ and $2 \mathrm{~cm}$.

For single cell simulations, a forward Euler scheme was used to solve the models with a time step of $\Delta t=0.002 \mathrm{~ms}$ for GPB, ORd and CRLP, and 

splitting scheme was used to solve the propagation (Heidenreich et al., 2010) with a space discretization of $\Delta x=0.1 \mathrm{~mm}$ and the same $\Delta t$ used in single cell simulations.

The results from single cell and in 1D tissue simulations were compared using the absolute $\left(E_{a}\right)$ and relative $\left(E_{r}\right)$ differences:

$$
\begin{gathered}
E_{a}=M_{j}^{\text {cell }}-M_{j}^{\text {tissue }} \\
E_{r}(\%)=\frac{M_{j}^{\text {cell }}-M_{j}^{\text {tissue }}}{M_{j}^{\text {tissue }}} \cdot 100
\end{gathered}
$$

where $M_{j}^{\text {cell }}$ and $M_{j}^{\text {tissue }}$ are the values of the physiological marker $j$ in cell and tissue for the condition under analysis.

\section{Results}

\subsection{Characterization of L-type calcium voltage-dependent inactivation}

Results of the paired-pulse protocol for steady-state voltage-dependent $I_{C a, L}$ inactivation are shown in Figure 2. Each model was compared with the set of experimental data that was used to characterize voltage-dependent $I_{C a, L}$ inactivation.

The TP06 model was able to reproduce well enough the experiments from Pelzmann et al. (1998) (see Figure 2a)). In this case, the modeled steady-state voltage-dependent $I_{C a, L}$ inactivation is the product of the steady-states values $\left(f_{2, s s}, f_{s s}\right)$ of the two inactivation gates $\left(f_{2}, f\right)$. The results from the in silico simulations, which account for additional interaction effects, were closer to the experimental data than the mathematical model. Figure 3 ,a) shows that for the TP06 model, the slow inactivation gate $f$ did not reach steady-state for high potentials $\left(V_{\text {pre }}=-5 \mathrm{mV}\right.$ and $\left.V_{\text {pre }}=40 \mathrm{mV}\right)$. Figure 3.a) also shows that the calcium-dependent inactivation gate has a less significant effect than the other gates, being this effect slightly more pronounced at lower potentials. Finally, one of the most significant differences between the model outcome (multiplication 
a)

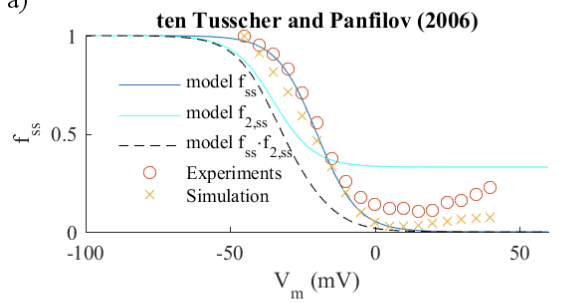

c)

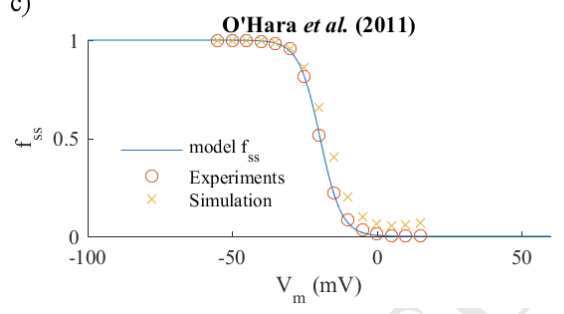

b)

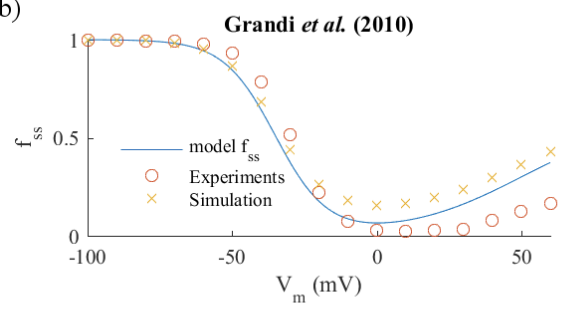

d)

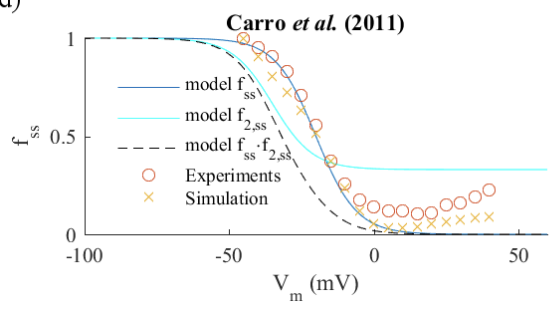

Figure 2: Comparison between model definition (continuous and discontinuous lines), in silico simulations (yellow crosses) and experimental results (red circles) for $f_{s s}$. a) TP06 model and data from Pelzmann et al. (1998). b) GPB model and data from Li et al. (1999). c) ORd model and data from Magyar et al. (2000). d) CRLP model and data from Pelzmann et al. (1998). 
a)
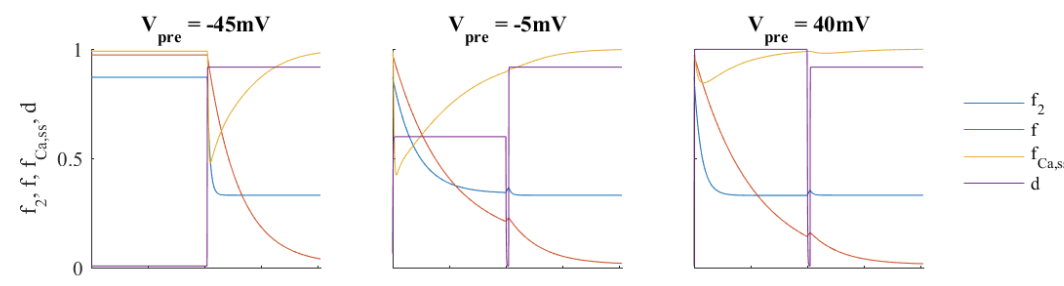

b)
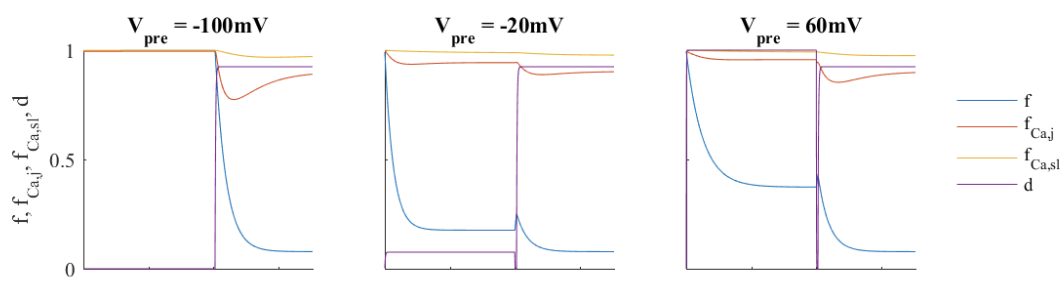

c)
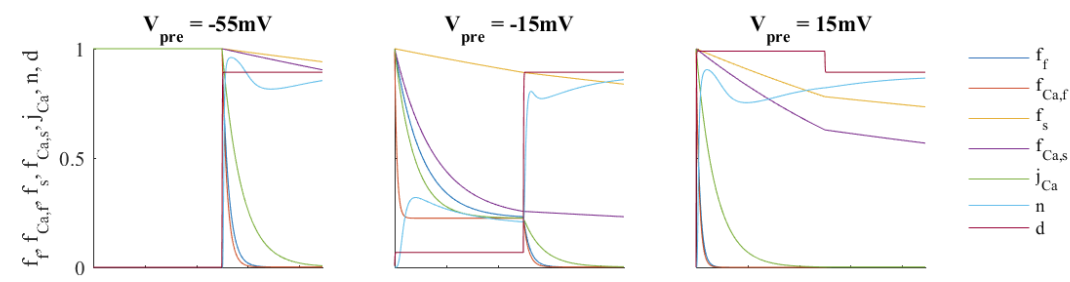

d)
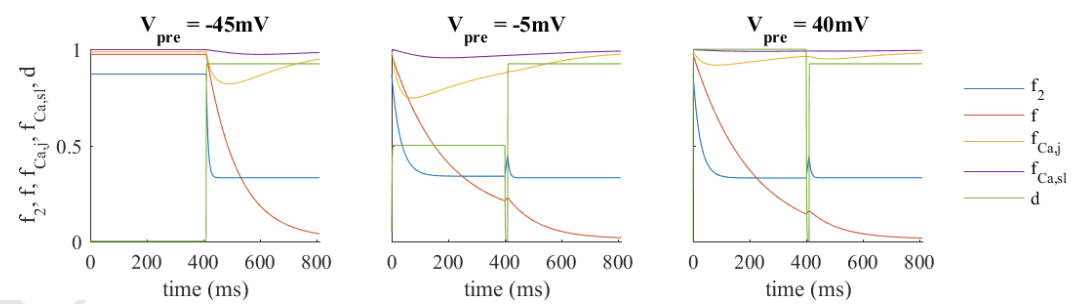

Figure 3: Temporal evolution of the $I_{C a, L}$ gates during the simulation of the paired-pulse protocol $\left(t_{\text {pre }}, t_{\text {sep }}\right.$ and $\left.t_{\text {pulse }}\right)$ : a) TP06 model. b) GPB model. c) ORd model. d) CRLP model 
of the steady-state values of the gates, black dashed line in Figure 2,a) and the result of the in silico simulations (yellow crosses) was that the curves were

formulation for the $I_{C a, L}$ inactivation. However, the difference between the results of the CRLP model and the experiments from Pelzmann et al. (1998) was smaller than for the TP06 model (see Figure 2/d)). 


\subsection{Ionic contributors to AP markers}

The results of the analyzed AP markers under control conditions are shown in Table 2 for the four analyzed models. Table 3 shows absolute and relative differences between cell and tissue values for each physiological marker. APD was very similar in cell and tissue simulations at $90 \%$ and $75 \%$ repolarization. The differences between cell and tissue increased at lower percentages of repolarization: the relative differences for APD at $50 \%$ repolarization were between $-0.7 \%$ and $-3.6 \%$ and considerably higher at $25 \%$ repolarization, between $4.9 \%$ and $37.4 \%$. The differences in Triangulation were mainly due to differences in $\mathrm{APD}_{50}$. Due to the smaller values of Triangulation, the relative differences for this AP marker were higher (between $3.0 \%$ and 28.8\%). The GPB model and the CRLP model were the ones that showed larger differences between cell and tissue results. On the contrary, the ORd model was the one showing less differences between cell and tissue in the AP-related electrophysiological markers.

The value of the resting potential $\left(V_{\min }\right)$ was the same in cell and tissue. On the contrary, the peak AP value $\left(V_{\max }\right)$ showed large differences between cell and tissue simulations (from $16.3 \%$ to $73.5 \%$ ). Similarly, large differences were quantified for $d V / d t_{\max }$ and $d V / d t_{\min }$. The ORd model showed the smallest differences in those voltage-related electrophysiological markers except for $d V / d t_{\max }$. The other three models showed similar values, in particular for the absolute differences.

For the TP06 model, the $I_{C a, L}$ current played different roles in cell and tissue simulations (see Figure 44). While in tissue it was the most important current to determine $V_{\max }$, in cell its effect on $V_{\max }$ was negligible. A similar effect was observed for the Triangulation, where $I_{C a, L}$ was the second current in order of relevance, but in cell simulations it had almost no effect on Triangulation. On the contrary, the impact of $I_{N a}$ current in cell were notably diminished in tissue simulations. For $V_{\max }$, the weight of the $I_{N a}$ current in cell was $51.6 \%$, while in tissue it was nearly $5 \%$. This also happened with the $I_{t o}$ current, although to a lesser extent. $G_{t o}$ conductance affected $d V / d t_{\min }$ in cell simulations, whereas in tissue simulations this marker was mainly controlled by $I_{C a, L}$. 


\begin{tabular}{|c|c|c|c|c|c|c|c|c|c|c|}
\hline & & P06 & & PB & $\mathrm{CF}$ & RLP & & $\mathrm{Rd}$ & OR & $\mathrm{dNa}$ \\
\hline & Cell & Fiber & Cell & Fiber & Cell & Fiber & Cell & Fiber & Cell & Fiber \\
\hline$A P D_{90}(\mathrm{~ms})$ & 300.5 & 300.2 & 286.1 & 283.5 & 306.2 & 306.7 & 224.3 & 224.2 & 223.2 & 224.0 \\
\hline$A P D_{75}(\mathrm{~ms})$ & 292.1 & 292.4 & 272.6 & 271.8 & 281.1 & 284.5 & 208.1 & 208.6 & 206.2 & 208.5 \\
\hline$A P D_{50}(\mathrm{~ms})$ & 272.4 & 277.0 & 235.0 & 243.8 & 228.1 & 239.6 & 177.8 & 179.1 & 174.4 & 179.2 \\
\hline$A P D_{25}(\mathrm{~ms})$ & 212.3 & 242.1 & 127.3 & 180.4 & 111.8 & 179.1 & 137.0 & 144.1 & 126.5 & 144.3 \\
\hline Trian. (ms) & 28.1 & 23.2 & 51.1 & 39.7 & 78.1 & 67.1 & 46.5 & 45.1 & 48.8 & 44.8 \\
\hline$V_{\max }(\mathrm{mV})$ & 38.5 & 24.6 & 38.7 & 23.4 & 38.1 & 21.9 & 36.2 & 31.1 & 41.9 & 30.9 \\
\hline$V_{\min }(\mathrm{mV})$ & -85.4 & -85.4 & -81.4 & -81.4 & -84.1 & -84.2 & -87.8 & -87.8 & -87.8 & -87.9 \\
\hline$\frac{d V}{d t} \max (\mathrm{V} / \mathrm{s})$ & 292.6 & 193.7 & 322.8 & 234.5 & 335.4 & 227.5 & 234.9 & 78.1 & 364.2 & 237.9 \\
\hline$\frac{d V}{d t} \min (\mathrm{V} / \mathrm{s})$ & -9.5 & -3.3 & -6.2 & -1.4 & -5.9 & -0.8 & -1.8 & -1.1 & -2.9 & -1.2 \\
\hline
\end{tabular}

Table 2: Comparison of electrophysiological markers simulated in single cell and 1D tissue under control conditions.

$E_{a} E_{r}(\%)$

TP06 GPB CRLP ORd ORdNa TP06 GPB CRLP ORd ORdNa

\begin{tabular}{|c|c|c|c|c|c|c|c|c|c|c|}
\hline$\overline{A P D_{90}}$ & 0.3 & 2.6 & -0.5 & 0.1 & $\overline{-0.8}$ & 0.1 & 0.9 & -0.2 & 0.0 & -0.3 \\
\hline$P D_{75}(\mathrm{~ms})$ & -0.3 & 8 & -3.4 & -0.5 & .3 & -0.1 & J & 1.2 & -0.2 & -1.1 \\
\hline$P D_{50}(\mathrm{~ms})$ & -4.6 & -8.8 & 1.5 & -1.3 & .8 & .7 & -3. & 1.8 & -0 & -2.7 \\
\hline$P D_{25}(\mathrm{~ms})$ & 20 & 53.9 & -67.3 & -7.1 & 17.8 & -12.3 & -29.4 & -37.6 & -4.9 & -12.3 \\
\hline rian. $(\mathrm{ms})$ & 4.9 & .4 & 11.0 & 1.4 & 4.0 & 20.7 & 28.8 & 16.4 & 3.0 & 9.0 \\
\hline $\operatorname{nax}(\mathrm{mV})$ & 13.9 & 15.3 & 16.2 & 5.1 & 11.0 & 56.4 & 65. & 73.5 & 16. & 35.5 \\
\hline $\min (\mathrm{mV})$ & 0.0 & 0.0 & 0.1 & 0.0 & 0.1 & 0.0 & 0.0 & 0.0 & 0.0 & 0.0 \\
\hline$\frac{d V}{d t} \max (\mathrm{V} / \mathrm{s})$ & 8.9 & 88.3 & 107.9 & 156.8 & 126.3 & 51.1 & 37.7 & 47.5 & 200. & 53.1 \\
\hline$\frac{d V}{d t} \min (\mathrm{V} / \mathrm{s})$ & -6.2 & -4.8 & -5.1 & -0.7 & -1.7 & 187. & 343.2 & 616.3 & 52.5 & 43.1 \\
\hline
\end{tabular}

Table 3: Absolute (Ea) and relative (Er) differences between electrophysiological markers in single cell and 1D tissue simulations. 


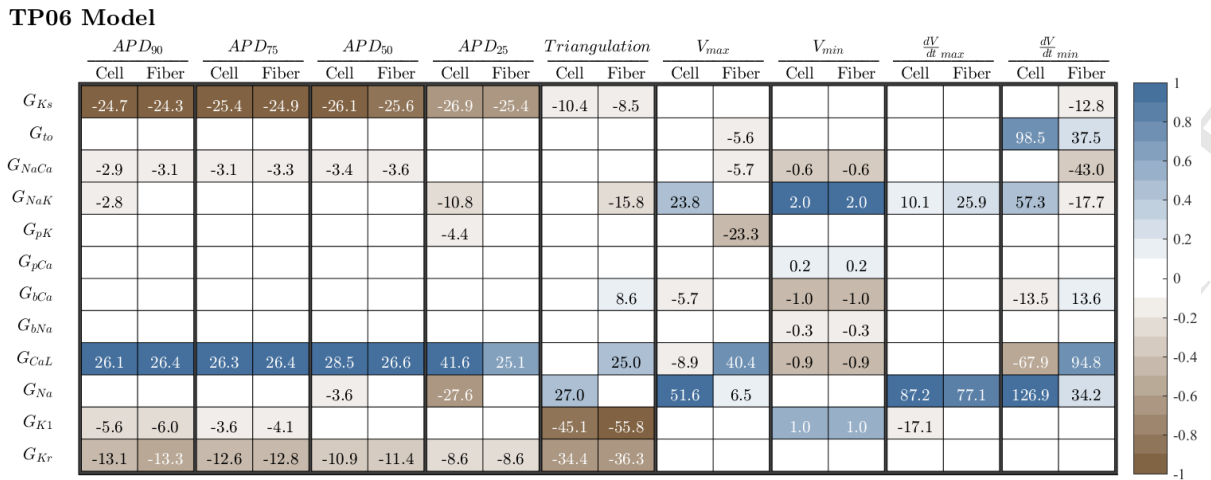

Figure 4: Contribution of ionic conductances to electrophysiological markers simulated in tissue and cell with the TP06 model. Dark colors (blue or brown) indicate maximum correlation between changes in a conductance and changes in a marker; white color indicates no correlation. Percentages in boxes indicate the contribution of changes in a conductance to changes in a marker. Minus signs indicate that conductances and markers vary inversely; plus signs indicate that conductances and markers vary in the same direction.

The more influential currents in the GPB model differed from the TP06 model (Figure 5). In this model, the $I_{C l, B k}$ current, which is not present in the TP06 model, was the most relevant contributor to APD in both cell and tissue simulations. Other effects were similar in both models. As an example, the $I_{N a}$ current contribution was reduced in the GPB model when the electrophysiological markers were calculated in tissue as compared to cell. This reduction was found for all markers except for $d V / d t_{\max }$. Similarly to the TP06 model, one current had a larger contribution to $V_{\max }$ in tissue than in cell, but in the GPB model that current was $I_{t o}$. The same behavior was found for Triangulation and $I_{K 1}$.

The APD of the ORd model was mainly controlled by $I_{K r}$ in cell, whereas in tissue the $I_{N a}$ also played an important role. The original ORd model showed large differences in the role played by the $I_{N a}$ current in tissue compared with the one in cell (Figure 6). In cell simulations, when the conductance of $I_{N a}$ increased, the APD at different percentages of repolarization decreased slightly. On the contrary, in tissue, when the conductance of the $I_{N a}$ increased, the APD 


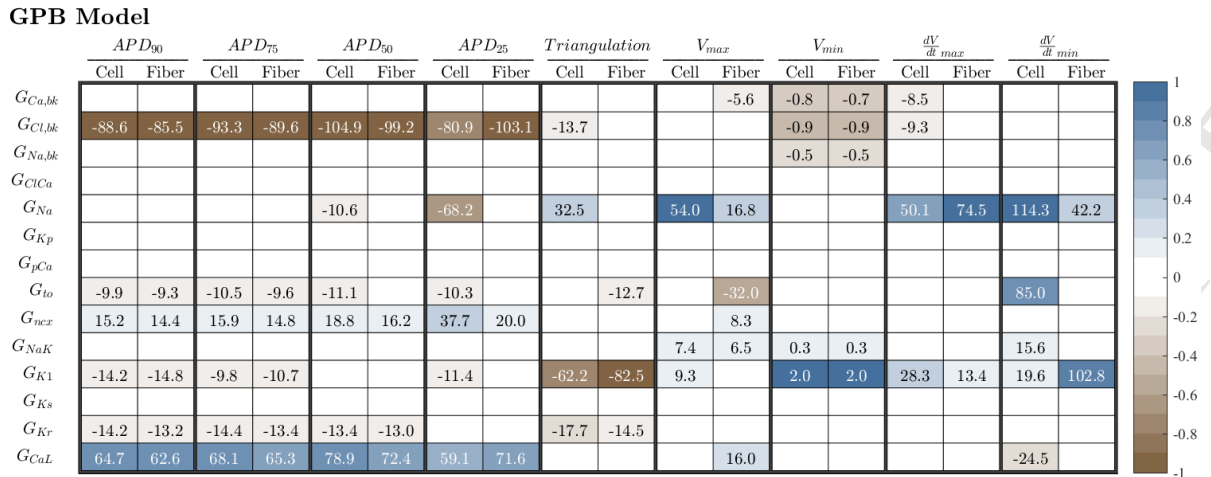

Figure 5: Contribution of ionic conductances to AP markers simulated in cell and tissue with the GPB model. The legend of the figure is the same as in see Figure 4

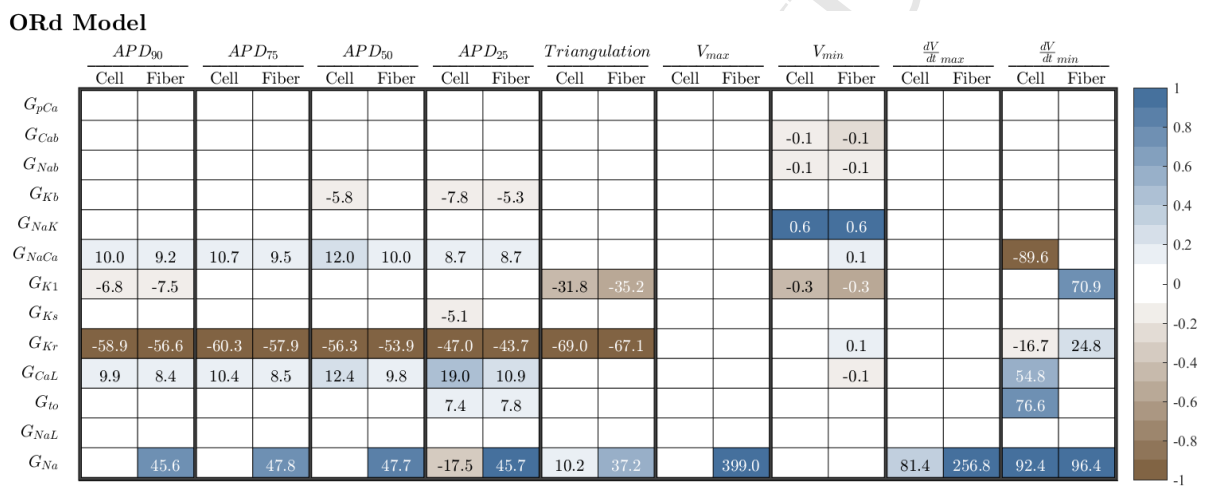

Figure 6: Contribution of ionic conductances to AP markers simulated in cell and tissue with the ORd model. The legend of the figure is the same as in see Figure 4

lengthened considerably. These differences in the ORd model disappeared when the $I_{N a}$ current formulation was replaced with the one described in the TP06 model. Also for $V_{\max }$, when $I_{N a}$ was replaced with the one defined in the TP06 model (Figure 7), the different behavior between cell and tissue simulations was interchanged.

The APD of the CRLP model highly depended on the conductance of the $I_{C l, B k}$ current (see Figure 8). This behavior is inherited from the GPB model but its weight in the CRLP model was found to be slightly smaller than in the GPB model. Likewise, the role of the $I_{C a, L}$ current was very similar to the 


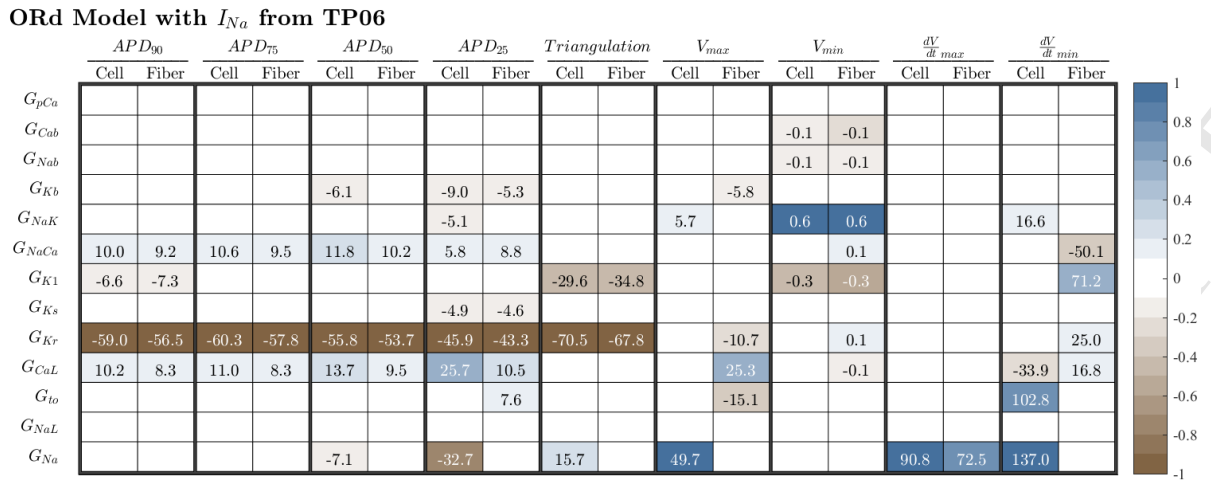

Figure 7: Contribution of ionic conductances to AP markers simulated in cell and tissue with the ORdNa model. The legend of the figure is the same as in see Figure 4

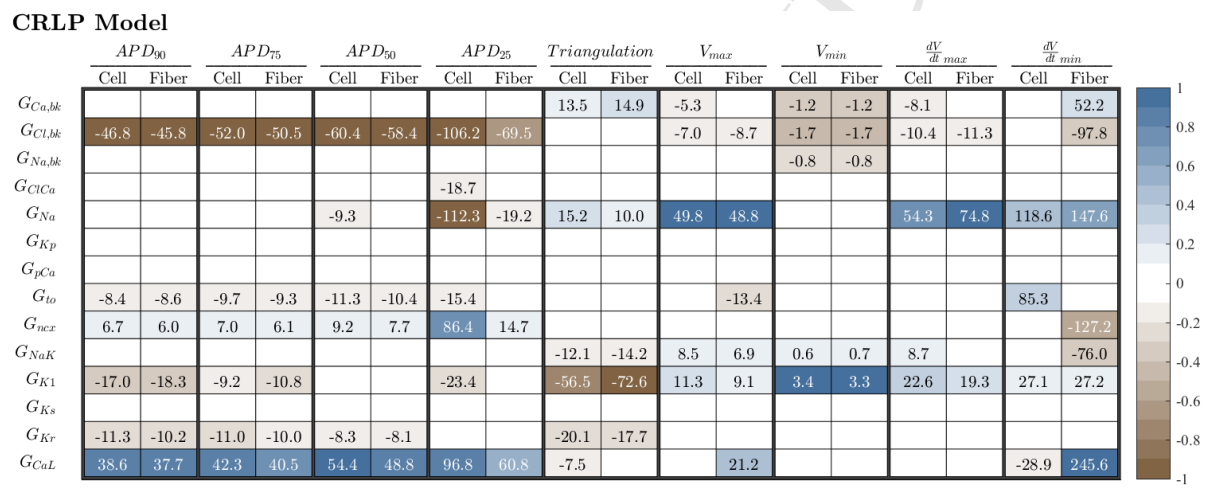

Figure 8: Contribution of ionic conductances to AP markers simulated in cell and tissue with the CRLP model. The legend of the figure is the same as in see Figure 4

\section{Discussion}

In this work we have analyzed issues arising during model development and validation, namely: i) the effect of submodel variable interactions in the char- 
acterization of ion channel gating, and ii) the effect of cell-to-cell interactions in the evaluation of ionic contributors to AP markers . A methodology for validating computational model formulations has been introduced. The proposed methodology consists in performing in silico simulations using the same protocol as in the experiments used to characterize a given current or AP marker. The methodology accounts for all variables involved in the model formulation as well as their interactions. It has been applied to evaluate voltage-dependent $I_{C a, L}$ inactivation and contributors to electrophysiological AP markers in four human ventricular models presenting different ionic formulations.

\subsection{Effect of the submodel variable interactions in the evaluation of voltage-} dependent L-Type Calcium Current inactivation

In the case of voltage-dependent $I_{C a, L}$ inactivation, our results show large differences between $I_{C a, L}$ inactivation as calculated from the model equation and $I_{C a, L}$ inactivation from the in silico simulations. Such differences were due to the interaction between voltage-dependent $I_{C a, L}$ inactivation gating and other effects such as $I_{C a, L}$ activation gating, protocol definition or duration of the voltage pulses used to calculate inactivation properties. This suggests that, when proposing any new model formulation, consistency between such formulation and the corresponding experimental data that is aimed at being reproduced needs to be first verified considering all involved factors.

In the TP06 model, the product of the steady-state values of the two voltagedependent $I_{C a, L}$ inactivation gates was very different from the experimental behavior. However, the results of the in silico simulations obtained with this model were in good agreement with the experiments. There are two effects that explain these results: i) normalization by $I_{C a, L}$ current peak at the minimum pre-pulse potential, as performed in the experimental protocol (Pelzmann et al. 1998), is done for a potential where the product of the two voltage-dependent inactivation gates is less than 1 ; ii) voltage-dependent inactivation gates do not reach a steady-state value at the end of the pre-pulse interval for some potentials. One of the most significant differences between the model outcome (multiplication of 
the steady-state values of the gates) and the result of the in silico simulations is that the curves representing the steady-state voltage-dependent inactivation (Figure 2 a)) were shifted. This shift made the in silico simulations approximate the experimental behavior due to the performed current peak normalization at a potential where inactivation probability is less than one.

The GPB model and the associated in silico simulations provided similar results to the experiments used to fit the steady-state value of voltage-dependent inactivation gating. Differences were mainly caused by the protocol used to measured $f_{s s}$, as explained in the following: the separation pulse had a different effect depending on the pre-pulse potential. For pre-pulse potentials similar to $V_{\text {hold }}$ the value of $f_{\text {ss }}$ obtained at $V_{\text {pulse }}$ is minimally affected since the steadystate values of $f$ for $V_{\text {hold }}$ and $V_{\text {pre }}$ are very similar. However, for larger values 385 of $V_{\text {pre }}$, and therefore larger differences between $V_{\text {hold }}$ and $V_{\text {pre }}$, the steadystate value of $f$ corresponding to $V_{\text {pre }}$ is very different to the steady-state value corresponding to $V_{\text {hold }}$, which corresponds to one. This causes that during the separation pulse the value of $f$ increases (see Figure 2,b)) and the difference in $f_{s s}$ between the model definition and the simulation increases with $V_{\text {pre }}$. In addition, the interaction with voltage-dependent activation, represented by gate $d$, always reduced the measured value, but the normalization, using the result of the minimum pre-pulse potential, nearly corrected this behavior. Finally, differences between experiments (Li et al., 1999) and in silico simulations get larger where differences between the model definition for $f_{s s}$ and experimental data are larger (see Figure 2.b) for positive potentials). These results suggest that a simple $I_{C a, L}$ inactivation formulation like that in the GPB model, with only one voltage-dependent inactivation gate, suffices to reproduce the experimental behavior of steady-state voltage-dependent $I_{C a, L}$ inactivation (Li et al. 1999 ).

In the ORd model, the definition of voltage-dependent $I_{C a, L}$ inactivation as a weighted sum of fast and slow inactivation gates is consistent with how experimentalists calculate inactivation time constants (O'Hara et al., 2011; Pelzmann et al. 1998). Time constants are calculated by fitting a biexponential function to the inactivation phase of the experimental current traces. However, impor- 
tant discrepancies between the ORd model simulations and the experiments

used to develop the model (Magyar et al. 2000) were found for pre-pulse potentials between $-20 \mathrm{mV}$ and $-10 \mathrm{mV}$. There are two possible causes behind such discrepancies: i) the time constant of the slow voltage-dependent inactivation gate; ii) the definition of voltage-dependent $I_{C a, L}$ inactivation as the sum of two gates. The first cause has to do with the fact that ORd model time constants are identified based on a simple-pulse test protocol of $75 \mathrm{~ms}$ duration, whereas the slow time constant of voltage-dependent $I_{C a, L}$ inactivation in the ORd model is of the order of 10 seconds. The slowness of the inactivation gate prevents reaching the steady-state value by the end of the pre-pulse, which overestimates the value of $f_{s s}$ at $V_{p u l s e}$. This is reinforced by the fact that voltage-dependent $I_{C a, L}$ inactivation is formulated as a sum of two gates. If inactivation is expressed as a product, as in the case of the TP06 model, when one of the gates reaches zero, the product reaches zero. However, for the ORd model the situation is more complex. Due to the interaction between the inactivation gates and the $n$ and $j_{C a}$ gates in this model, as shown in section 3.1 , the result for potentials above $-10 \mathrm{mV}$ is equivalent to the product of two gates since the $j_{C a}$ gate is almost zero, and therefore the result from the simulation is similar to the model formulation of $f_{s s}$ and experiments. On the contrary, for potentials between -20 $\mathrm{mV}$ and $-10 \mathrm{mV}$, where by the end of the pre-pulse the $j_{C a}$ gate is far from zero and the slow inactivation gates, in particular $f_{C a, s}$, are far from reaching their steady-state value, the $f_{s s}$ value obtained from the simulation is considerably larger than the $f_{s s}$ value defined in the model formulation and the experiments.

For the CRLP model, similarly to the TP06 model, the largest differences between model equations, in silico simulations and experiments are due to the time constants of the activation and inactivation gates. Nevertheless, the results of the in silico simulations with this model reproduced the experimental observations slightly better than the TP06 model. In the CRLP model, the modeled steady-state voltage-dependent $I_{C a, L}$ inactivation has a faster gate $f_{2}$ than the TP06 model (as defined by the time constant $\tau_{f 2}$ ). Due to this faster gate, the effect of the separation pulse was larger than in the TP06 model because during 
As a consequence, the CRLP model yielded a larger $f_{s s}$ value in the in silico measurements, which was closer to the experimental values. This effect was not very pronounced but it could be better observed for voltages where $f_{2}$ is larger (around $-20 \mathrm{mV}$ ).

440

In this work we have focused on the difficulties of fitting model parameters associated with the steady-state value of $I_{C a, L}$ inactivation using data obtained from voltage-clamp experiments. Such difficulties arise from submodel variable interactions in the $I_{C a, L}$ formulation. This situation is a common problem to many gating variables in cardiac electrophysiological models. In this regard, most experimental protocols cannot measure the behavior of one gate at a time, instead, they measure the behavior of all gates of the same nature (activation, inactivation, etc.) all together (e.g. for $I_{C a, L}$ inactivation the experimental protocol measures fast and slow inactivation together). Therefore the model needs to consider the coexistence of all gates during parameter identification in order to accurately reproduce the experimental results. This issues are also present in the estimation of the time constants associated with the different gates. In this regard, the gating interactions occurring in an experiment (due to the particular set of experimental parameters) implies that the identified time constants associated with the experimental data cannot, in general, be associated with an individual gate of the model.

Some authors have tried to take into account how gating interactions affect the results of the voltage-clamp protocol (Lee et al., 2006; Wang and Beaumont, 2004). The voltage-clamp protocol is not designed to measure this directly, but the corresponding effects are included in the experimental results. Nevertheless, most of these techniques have been developed for currents with only one inactivation and one activation gate. How to extend those methods in order to better calibrate complex gate combinations remains to be investigated. 
4.2. Effect of cell-to-cell interactions in the evaluation of ionic contributors to AP markers

While the ionic currents with the largest effects over each AP marker varied from one model to another, the differences between single cell and tissue simulations were comparable in all the models for all the analyzed markers.

As expected, measurement of depolarization-related AP markers in single cell simulations was significantly affected by the externally applied stimulation current. In particular, $d V / d t_{\max }$ in single cell simulations was quite different from that obtained in tissue for all considered models. Although some models use $d V / d t_{\max }$ or $V_{\max }$ measured in single cell simulations to adjust $I_{N a}$ Courtemanche et al., 1998, Grandi et al., 2010; O'Hara et al., 2011), our results suggest that $I_{N a}$ should be adjusted with the results obtained from, at least, a simulation in 1D tissue as in Carro et al. (2011), especially if the experiments were performed in tissue.

For repolarization-related AP markers, the largest differences between cell and tissue were observed for Triangulation and $\mathrm{APD}_{25}$. The results for $\mathrm{APD}_{90}$ and $\mathrm{APD}_{75}$ were very consistent between cell and tissue, while a small discrepancy was found for $\mathrm{APD}_{50}$. Although differences for $\mathrm{APD}_{9} 0$ and $\mathrm{APD}_{50}$ were small, their added contribution together with the small value of Triangulation caused a relatively large accumulated difference for this marker. For this reason, Triangulation should be used with caution as a marker in single cell simulations when used to calibrate the AP model.

Importantly, the differences between markers computed from single cell and 1D tissue simulations were not only the reflect of a constant bias, as shown in Table 2 and in Table 3 . For repolarization markers, those differences reflected the different contribution of ionic currents to the markers. Computational studies using single cell simulations to analyze the sensitivity of different ionic current conductances on the repolarization behavior of the model should only consider markers with a validated correspondence between cell and tissue simulations, or otherwise use markers computed from tissue rather than from single cell simulations. 


\section{Conclusions}

495

When proposing a new model, or when evaluating an existing model, consistency between simulated and experimental data should be verified considering all involved effects and scales. The closer the experimental conditions are reproduced in the computer simulations, the more robust the process of model development and validation will be.

As discussed in this paper, proper characterization and validation of a given model should be performed with the in silico simulation of the experimental protocol. In this way, the effects of the interaction between model variables are accounted for and modelling inconsistencies are avoided.

While the information provided by electrophysiological markers is very valuable for model development and validation, markers should be computed using simulations that resemble as closely as possible the conditions used for the experimental measurements, or, at least, the consistency of the marker in the different scales should be previously validated to avoid misunderstandings.

As a final remark, complex models represent a real challenge for parameter identification and validation. This does not mean that models should be necessarily simple, but that complex models require additional testing in order to fully verify their correct performance.

\section{Acknowledgements}

This work was supported by Ministerio de Economía y Competitividad and FEDER (EU), under projects TIN2012-37546-C03-03 and TIN2013-41998-R, by CIBER in Bioengineering, Biomaterials \& Nanomedicine (CIBER-BBN) through Instituto de Salud Carlos III, and by Grupo Consolidado BSICoS (T96) and Grupo Consolidado AMB (T88), from DGA and European Social Fund. The computation was performed using the ICTS "NANBIOSIS", more specifi-

${ }_{520}$ cally the High Performance Computing Unit of CIBER-BBN at the University of Zaragoza. 


\section{References}

Carmeliet, E., 1999. Cardiac ionic currents and acute ischemia: from channels to arrhythmias. Physiol Rev 79, 917-1017. URL: http://physrev.physiology . org/content/79/3/917.

Carro, J., Rodríguez, J., Laguna, P., Pueyo, E., 2011. A human ventricular cell model for investigation of cardiac arrhythmias under hyperkalaemic conditions. Philos T Roy Soc A 369, 4205-4232. doi:10.1098/rsta.2011.0127

Cherry, E.M., Fenton, F.H., 2007. A tale of two dogs: analyzing two models of canine ventricular electrophysiology. Am J Physiol Heart Circ Physiol 292, H43-H55. doi 10.1152/ajpheart.00955. 2006

Courtemanche, M., Ramirez, R.J., Nattel, S., 1998. Ionic mechanisms underlying human atrial action potential properties: insights from a mathematical model. Am J of Physiol 275, H301-H321. URL: http://ajpheart. physiology.org/content/275/1/H301

Csercsik, D., Hangos, K.M., Szederkényi, G., 2012. Identifiability analysis and parameter estimation of a single Hodgkin-Huxley type voltage dependent ion channel under voltage step measurement conditions. Neurocomputing 77, 178-188. doi:10.1016/j.neucom.2011.09.006.

Dokos, S., Lovell, N.H., 2004. Parameter estimation in cardiac ionic models, in:

a Prog Biophys Mol Bio, pp. 407-431. doi 10.1016/j.pbiomolbio.2004.02. 002

Grandi, E., Pasqualini, F.S., Bers, D.M., 2010. A novel computational model of the human ventricular action potential and Ca transient. J Mol Cell Cardiol 48, 112-121. doi $10.1016 /$ j.yjmcc.2009.09.019.

Heidenreich, E., Ferrero, J.M., Doblaré, M., Rodríguez, J.F., 2010. Adaptive macro finite elements for the numerical solution of monodomain equations

口 in cardiac electrophysiology. Ann Biomed Eng 38, 2331-2345. doi 10.1007/ s10439-010-9997-2. 

of the action potential predict serious proarrhythmia, but action potential

Lee, J., Smaill, B., Smith, N., 2006. Hodgkin-Huxley type ion channel characterization: An improved method of voltage clamp experiment parameter estimation. J Theor Biol 242, 123-134. doi 10.1016/j·jtbi.2006.02.006.

Li, G.R., Nattel, S., 1997. Properties of human atrial $I_{C a}$ at physiological temperatures and relevance to action potential. Am J Physiol Heart Circ Physiol

\ 272, H227-H235. URL: http://ajpheart.physiology.org/content/272/ $1 / \mathrm{H} 227$.

Li, G.R., Yang, B., Feng, J., Bosch, R.F., Carrier, M., Nattel, S., 1999. Transmembrane $I_{C a}$ contributes to rate-dependent changes of action potentials in human ventricular myocytes. Am J Physiol Heart Circ Physiol 276, H98-106. URL: http: //ajpheart.physiology .org/content/276/1/H98.

Magyar, J., Iost, N., Körtvély, Á., Bányász, T., Virág, L., Szigligeti, P., Varró, A., Opincariu, M., Szécsi, J., Papp, J.G., Nánási, P.P., 2000. Effects of endothelin-1 on calcium and potassium currents in undiseased human ventricular myocytes. Pflügers Arch 441, 144-149. doi:10.1007/s004240000400.

Magyar, J., Szentandrássy, N., Bányász, T., Fülöp, L., Varró, A., Nánási, P.P., 2002. Effects of thymol on calcium and potassium currents in canine and q human ventricular cardiomyocytes. Brit J Pharmacol 136, 330-338. doi:10. 1038/sj.bjp.0704718.

Noble, D., Garny, A., Noble, P.J., 2012. How the Hodgkin-Huxley equations

घ inspired the Cardiac Physiome Project. J Physiol 590, 2613-2628. doi 10. 1113/jphysiol.2011.224238.

O’Hara, T., Virág, L., Varró, A., Rudy, Y., 2011. Simulation of the undiseased human cardiac ventricular action potential: Model formulation and 
₫ experimental validation. PLOS Comput Bio 7. doi 10.1371/journal.pcbi. 1002061

Taggart, P., Sutton, P., T, O., Coronel, R., Trimlett, R., Pugsley, W., 2000. Inhomogeneous transmural conduction during early ischemia in patients with

q ventricular tissue. Am J Physiol Heart Circ Physiol 286, H1573-1589. doi-10. 1152/ajpheart.00794.2003 
ten Tusscher, K., Panfilov, A., 2006. Alternans and spiral breakup in a human ventricular tissue model. Am J Physiol Heart Circ Physiol 291, H1088-H1100. doi:10.1152/ajpheart.00109.2006.

Volders, P.G., Vos, M.A., Szabo, B., Sipido, K.R., de Groot, S.H., Gorgels, A.P., Wellens, H.J., Lazzara, R., 2000. Progress in the understanding of cardiac early afterdepolarizations and torsades de pointes: time to revise

current concepts. Cardiovasc Res 46, 376-392. doi 10.1016/S0008-6363(00) $00022-5$

Wang, G.J., Beaumont, J., 2004. Parameter estimation of the hodgkin-huxley gating model: An inversion procedure. Siam J Appl Math 64, 1249-1267. doi:10.1137/S0036139902419826.

${ }_{615}$ Yue, L., Feng, J., Li, G.R., Nattel, S., 1996. Transient outward and delayed rectifier currents in canine atrium: properties and role of isolation methods. Am

口 J Physiol 270, H2157-68. URL: http://wWW.ncbi.nlm.nih.gov/pubmed/ 8764269 .

Zeng, J., Laurita, K.R., Rosenbaum, D.S., Rudy, Y., 1995. Two components of the delayed rectifier $\mathrm{K}+$ current in ventricular myocytes of the guinea pig type. Theoretical formulation and their role in repolarization. Circ Res 77, 140-152. doi $10.1161 / 01$.RES.77.1.140. 
Corrigendum

Corrigendum to <' Limitations in electrophysiological model development and validation caused by differences between simulations and experimental protocols'> $<$ [Progress in Biophysics and Molecular Biology 129 (2017) 5364]>

$<$ Jesús Carro a, b, c, *, José F. Rodríguez-Matas ${ }^{\text {b, d, }}{ }^{\text {, Violeta }}$ Monasterio a ${ }^{\text {, Esther Pueyo }}{ }^{\text {b, }}{ }_{>}$ $<$ a Universidad San Jorge, Campus Universitario, Autov A23 Km 299, 50830, Villanueva de Gállego, Zaragoza, Spain b Aragon Institute for Engineering Research, Universidad de Zaragoza, Spain

${ }^{\mathrm{c}}$ CIBER in Bioengineering, Biomaterials \& Nanomedicine (CIBER-BBN), Spain

${ }^{\mathrm{d}}$ LaBS, Department of Chemistry, Materials and Chemical Engineering “Giulio Natta”, Politecnico di Milano, Italy>

The authors regret $<$

Acknowledgments should read as follows:

This work was supported by Ministerio de Economía y Competitividad and FEDER, under projects TIN2012-37546-C03-03 and TIN2013-41998-R, by the European Research Council (ERC) through project ERC-2014-StG 638284 to EP, by CIBER in Bioengineering, Biomaterials \& Nanomedicine (CIBER-BBN) through Instituto de Salud Carlos III, and by Grupo Consolidado BSICoS (T96) and Grupo Consolidado AMB (T88), from DGA and European Social Fund. The computation was performed using the ICTS "NANBIOSIS", more specifically the High Performance Computing Unit of CIBER-BBN at the University of Zaragoza.

$>$.

The authors would like to apologise for any inconvenience caused.

DOI of original article: $<10.1016 / j$.pbiomolbio.2016.11.006>

< Jesús Carro. Universidad San Jorge, Campus Universitario, Autov A23 km 299, 50830, Villanueva de Gállego Zaragoza, Spain.>

<jcarro@usj.es> 